\title{
Coherent-interface-induced strain in large lattice-mismatched materials: A new approach for modeling Raman shift
}

\author{
Andrian V. Kuchuk ${ }^{1}(\bowtie)$, Fernando M. de Oliveira ${ }^{1,2}$, Pijush K. Ghosh ${ }^{3}$, Yuriy I. Mazur ${ }^{1}$, Hryhorii V. Stanchu ${ }^{1}$, \\ Marcio D. Teodoro ${ }^{2}$, Morgan E. Ware ${ }^{1,3}$, and Gregory J. Salamo ${ }^{1}$ \\ ${ }^{1}$ Institute for Nanoscience and Engineering, University of Arkansas, Fayetteville, AR 72701, USA \\ ${ }^{2}$ Departamento de Física, Universidade Federal de São Carlos, São Carlos, SP 13565-905, Brazil \\ ${ }^{3}$ Department of Electrical Engineering, University of Arkansas, Fayetteville, AR 72701, USA
}

(c) Tsinghua University Press and Springer-Verlag GmbH Germany, part of Springer Nature 2021

Received: 1 April 2021 / Revised: 17 August 2021 / Accepted: 31 August 2021

\begin{abstract}
Strain engineering as one of the most powerful techniques for tuning optical and electronic properties of III-nitrides requires reliable methods for strain investigation. In this work, we reveal, that the linear model based on the experimental data limited to within a small range of biaxial strains $(<0.2 \%)$, which is widely used for the non-destructive Raman study of strain with nanometer-scale spatial resolution is not valid for the binary wurtzite-structure group-III nitrides GaN and AIN. Importantly, we found that the discrepancy between the experimental values of strain and those calculated via Raman spectroscopy increases as the strain in both GaN and AIN increases. Herein, a new model has been developed to describe the strain-induced Raman frequency shift in GaN and AIN for a wide range of biaxial strains (up to 2.5\%). Finally, we proposed a new approach to correlate the Raman frequency shift and strain, which is based on the lattice coherency in the epitaxial layers of superlattice structures and can be used for a wide range of materials.
\end{abstract}

\section{KEYWORDS}

Raman spectroscopy, strain, lattice coherency, III-nitrides, high-resolution X-ray diffraction (HRXRD)

\section{Introduction}

Research in wide-bandgap III-nitride semiconductors continues to progress rapidly, especially for the materials with bandgap exceeding that of gallium nitride $(3.4 \mathrm{eV})$. Specifically, III-nitride based heterostructures provide new opportunities for a wide range of research and device applications, such as piezotronics and piezophototronics [1], self-powered photoelectrochemical-type photodetectors [2], room-temperature quantum emitters [3], single photon emitters [4-6], resonant tunneling diodes [7], highelectron-mobility transistors $[8,9]$, efficient photoelectrocatalysts for solar water splitting [10], multi-wavelength light-emitting diodes (LEDs) [11,12], and deep ultraviolet (DUV)-LEDs [13-17]. AlGaN-based DUV-LEDs represent a sustainable alternative to replace the environmentally harmful conventional mercury lamps [18] and thus, are becoming crucial for many applications such as water purification and/or inactivation of microorganisms, including bacteria, fungi, and viruses $[19,20]$.

Polarization, resulting from a lack of inversion symmetry between the anion and cation positions in the wurtzite crystal structure, has a dramatic impact on the optical and electronic properties of polar III-nitrides. The quantum-confined Stark effect, which is undesirable for LEDs is caused by the polarizationinduced internal electric field and reduces emission efficiency by separating the electron and hole wavefunctions within quantum wells (QWs) [21,22]. At the same time, proper engineering of both spontaneous and piezo-polarization fields permits the intentional and favorable tuning of the electronic properties of IIInitrides. For example, the activation of impurity dopants by the polarization charge in compositionally graded AlGaN semiconductors found very receptive applications in the fabrication of efficient DUV-LEDs and p-n junctions [23-27]. This polarization doping depends intimately on biaxial strain (piezo-polarization) and alloy composition (spontaneous polarization). Composition and strain are interrelated due to the in-plane lattice mismatch of $2.5 \%$ between GaN and AlN. Moreover, the misfit strain in lattice-mismatched epitaxial structures can relax by the creation of a high density of structural defects. Therefore, the tuning and analysis of strain in III-nitrides is an important task.

Raman spectroscopy and X-ray diffraction (XRD) are powerful methods for reliable and non-destructive analysis of strain in heterostructured materials [28-39]. One big advantage of Raman spectroscopy over the laboratory-based XRD technique is the ability to spatially resolved assessment of inhomogeneities in strain distribution at the nanoscale. Nanofocused scanning XRD with high spatial resolution can be measured only by using a synchrotron source [38]. Therefore, Raman spectroscopy has been effectively used to study strain-induced band gap engineering in $\mathrm{GaN} / \mathrm{Al}(\mathrm{Ga}) \mathrm{N}$ core-shell nanowire heterostructures [31], energy transport in $\mathrm{Ga}_{0.84} \mathrm{In}_{0.16} \mathrm{~N} / \mathrm{GaN}$ heterostructures [32], the strain state of ultrathin GaN QWs embedded in an AlN matrix [33], packaging-induced stress in blue GaN-LEDs soldered onto copper substrates [34], spatial non-uniformity in heavily Si- 
doped GaN layers [35], and phonon modes and in-plane strain $\left(\varepsilon_{x x}\right)$ in GaN/AlN multi-QW structures [37, 39]. However, a wide range of values of the deformation potential constants and elastic constants of $\mathrm{Al}(\mathrm{Ga}) \mathrm{N}$ used for calculation of strain via Raman spectroscopy lead to an uncertainty in the strain values. Moreover, the previous model in the literature for calculating strain from Raman data was based on experimental data limited to a small range of biaxial strains $\left(0 \%<\varepsilon_{x x}<0.2 \%\right)[34,40]$. Consequently, a disagreement between strain values calculated via Raman spectroscopy and from other methods is often found in the literature, especially for high biaxial strains $\left(\varepsilon_{x x}>0.2 \%\right)[33,39]$.

In this paper, we report on Raman spectroscopy of biaxial strain in GaN and AlN for a wide range of strains $\left(0 \%<\varepsilon_{x x}<2.5 \%\right)$. We have found that for both GaN and AlN the strain-induced Raman frequency shift falls out of the prediction of the linear model based on small biaxial strain values. Moreover, the discrepancy between Raman experimental data and the linear model increases as the strain in the GaN or AlN increases. In this work, a new approach is described that is applicable to study the biaxial strain in GaN(AlN) by Raman spectroscopy over a wide range of strains. The Raman frequency shift for GaN and AlN in a series of 30period GaN/AlN superlattices (SLs) with different thickness ratios $\left(t_{\mathrm{GaN}} / t_{\mathrm{AIN}}\right)$ was correlated with the strain from high-resolution XRD (HRXRD). A new model that describes well the experimental data and does not take into account the deformation potential constants and elastic constants of $\mathrm{GaN}(\mathrm{AlN})$ was introduced. Moreover, a lateral strain distribution in $\mathrm{GaN}$ and AlN layers in the close vicinity of a crack as a result of strain relaxation in GaN/AlN SL structures via Raman spectroscopy is demonstrated. Finally, we show that the lattice coherency between the layers in the SL structures can be used to correctly calculate the Raman frequency shift vs. strain in many other materials.

\section{Experimental details}

The GaN/AlN SLs were grown on GaN-on-sapphire templates by plasma assisted molecular beam epitaxy (PAMBE). The templates consist of $\sim 3 \mu \mathrm{m}$ thick [0001] oriented, unintentional doped GaN grown on $\sim 100 \mathrm{~nm}$ thick $\mathrm{AlN}-\mathrm{on}-\mathrm{Al}_{2} \mathrm{O}_{3}$ by hydride vapor phase epitaxy. Before growth, $1 \mu \mathrm{m}$ of titanium was deposited on the back side in order to improve the radiative heating of the substrate during growth. Then the substrate was cleaned using sonication in acetone, methanol, and isopropanol. After that, the sample was introduced into the vacuum system through a load-lock and baked at $200{ }^{\circ} \mathrm{C}$ for an hour with a base pressure of $\sim 5.0 \times 10^{-8}$ Torr. This is primarily to remove water vapor. Then the sample was transferred to an outgas chamber with base pressure of $\sim 5.0$ $\times 10^{-10}$ Torr, where the sample was degassed at $300{ }^{\circ} \mathrm{C}$ for an hour to further remove surface contaminants. Finally, the sample was transferred to the main growth chamber with a base pressure of $1.0 \times 10^{-11}$ Torr. Here the sample underwent a final heat clean at $830^{\circ} \mathrm{C}$ for an hour. This not only prepares the substrate surface for growth, but it also effectively outgasses the titanium backside coating so that the efficiency of the heating of the substrate remains constant throughout the growth.

A $~ 180 \mathrm{~nm}$ unintentionally doped GaN buffer layer was grown on the substrate with a growth rate $\sim 3 \mathrm{~nm} / \mathrm{min}$. The nitrogen plasma was maintained at $350 \mathrm{~W}$ with a $0.5 \mathrm{sccm}$ flow rate at a substrate temperature $\sim 795^{\circ} \mathrm{C}$. The chamber pressure during the growth was $\sim 2.65 \times 10^{-6}$ Torr. After that, a 30 period AlN/GaN superlattice was grown at the same temperature. The different SLto-substrate average lattice misfits, and consequently the strain of the GaN and AlN SL layers, were achieved by reducing the GaN QW thickness $\sim 7 \mathrm{~nm}$ (sample S1), $\sim 5 \mathrm{~nm}$ (sample S2), $\sim 4 \mathrm{~nm}$ (sample S3), $3 \mathrm{~nm}$ (sample S4), and $\sim 1 \mathrm{~nm}$ (sample S5), maintaining the thickness of the AlN barrier layers of about $3 \mathrm{~nm}$ for all samples. After growth, HRXRD measurements were performed using a Panalytical X'Pert Pro MRD diffractometer equipped with a $1.6 \mathrm{~kW} \mathrm{Cu} \mathrm{K \alpha} \alpha_{1} \mathrm{X}$-ray tube (vertical line focus), a standard four-bounce $\mathrm{Ge}(220)$ monochromator, and a Pixel detector. And, micro-Raman measurements were performed at room temperature in backscattering geometry, i.e., $z(x, x) \bar{z}+z(x, y) \bar{z}$ using Porto's notation using a $632.8 \mathrm{~nm} \mathrm{He}-$ Ne laser (Melles Griot) with $\sim 5 \mathrm{~mW}$ at the sample surface. The optical signal was collected by a $0.75 \mathrm{~m}$ long spectrometer (Horiba Jobin-Yvon LabRam HR800) equipped with a thermoelectrically cooled Si charge-coupled device (CCD) camera. The spot of the incident laser light was adjusted by the focus controllers of a microscope (Olympus BX41) using an objective lens with 100x magnification (Olympus MPLanN100x 0.9NA) and a digital camera (uEye), resulting in a $\sim 1 \mu \mathrm{m}$ diameter excitation spot. The light of a high-power lamp (Euromex Holland EK-1) was employed for acquiring images of the samples surface.

\section{Results and discussion}

A variety of phonon modes can be identified by Raman spectroscopy from the binary wurtzite-structure group-III nitrides [41-44]. However, the high energy mode $E_{2}^{\mathrm{H}}$ (TO-like) has been extensively used to study the strain properties in GaN and AlN. This is due to the fact that the nonpolar $E_{2}^{\mathrm{H}}$ mode represents transverse lattice oscillations propagating in the [0001] directions with atomic displacement of the nitrogen $(\mathrm{N})$ atoms alone within the (0001) plane (the $c$-plane) in the lattice of III-nitride materials. Therefore, the $E_{2}^{\mathrm{H}}$ mode in the wurtzite crystal structure is very sensitive to lateral strain with the largest strain dependent shift in energy. Representative Raman spectra of GaN/AlN SL grown on GaN-on-sapphire substrate is shown in Fig. 1.

Both the GaN and AlN layers from the substrate (template) are considered mostly relaxed with only slight amounts of residual stress. The $E_{2}^{\mathrm{H}}$ phonon modes labeled $\mathrm{GaN}_{\text {substr. }}$ and $\mathrm{AlN}_{\text {substr. }}$ are from these layers. The $E_{2}^{\mathrm{H}}\left(\mathrm{GaN}_{\text {substr. }}\right)$ at $568.6 \mathrm{~cm}^{-1}$ is slightly shifted from bulk $\mathrm{GaN}, E_{2}^{\mathrm{H}}\left(\mathrm{GaN}_{\text {bulk }}\right)$ at $567.4 \mathrm{~cm}^{-1}$ [8], whereas the $E_{2}^{\mathrm{H}}\left(\mathrm{AlN}_{\text {substr. }}\right)$ at $658 \mathrm{~cm}^{-1}$ is nearly identical with that of bulk AlN, $E_{2}^{\mathrm{H}}\left(\mathrm{AlN}_{\text {bulk }}\right)$ at $656.7 \pm 2.4 \mathrm{~cm}^{-1}[45-52]$. The $E_{2}^{\mathrm{H}}$ shift to the higher frequency in both cases indicates compressive residual stress in the template layers [33]. In contrast, a significant shift of the $E_{2}^{\mathrm{H}}$ is observed for both the GaN and AlN SL layers, which indicates that the SL structure is not pseudomorphic to the underlying

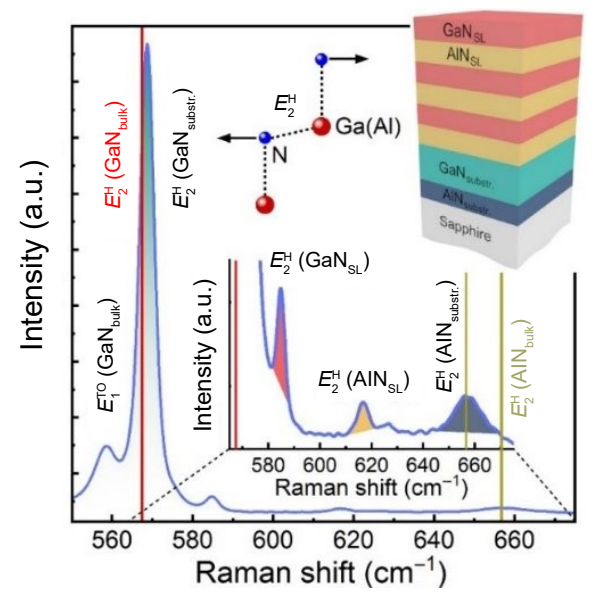

Figure 1 Raman spectra of GaN/AlN SL measured in the backscattering $z(x, x) \bar{z}+z(x, y) \bar{z}$ geometry. The phonon modes from the GaN and AlN in the substrate and the SL layers are labeled, along with the bulk relaxed values. The inset shows the Raman $E_{2}^{\mathrm{H}}$ phonon mode for $\mathrm{Ga}(\mathrm{Al}) \mathrm{N}$ in terms of the relative atomic vibrations. 
templates. Therefore, as a consequence of strain relaxation, the compressive strain in the GaN QWs leads to the shift of the $E_{2}^{\mathrm{H}}\left(\mathrm{GaN}_{\mathrm{SL}}\right)$ to higher frequency, while the $E_{2}^{\mathrm{H}}\left(\mathrm{AlN}_{\mathrm{SL}}\right)$ at lower frequency is due to tensile strain in the AlN SL layers. The magnitude of the shift $\left(\Delta \omega_{E_{2}^{\mathrm{H}}}\right)$ can be used for the strain calculation. Using Raman spectroscopy, the biaxial strain $\left(\varepsilon_{x x}=\varepsilon_{y y}\right)$ is usually calculated through two deformation potential constants $a_{E_{2}^{H}}$ and $b_{E_{2}^{\mathrm{H}}}$

$$
\Delta \omega_{E_{2}^{\mathrm{H}}}=2 a_{E_{2}^{\mathrm{H}}} \varepsilon_{x x}+b_{E_{2}^{\mathrm{H}}} \varepsilon_{z z}=\left(2 a_{E_{2}^{H}}+b_{E_{2}^{\mathrm{H}}} q\right) \varepsilon_{x x}=K \varepsilon_{x x}
$$

where $K=2 a_{E_{2}^{\mathrm{H}}}+b_{E_{2}^{\mathrm{H}}} q$ is a constant with, $q=\frac{\varepsilon_{z z}}{\varepsilon_{x x}}=-2 C_{13} / C_{33}$, the Poisson ratio and $C_{13}$ and $C_{33}$ elements of the elastic constant tensor $[33,39,40,44]$. Unfortunately, there are wide ranges of the values of $a_{E_{2}^{\mathrm{H}}}, b_{E_{2}^{\mathrm{H}}}, C_{13}$ and $C_{33}$ found in the literature, with averages and standard deviations of common values summarized in Table 1 (also see Table S1 in the Electronic Supplementary Material (ESM)). As a result, the strain value calculated from Raman spectroscopy depends strongly on the constants used in Eq. (1) where ultimately it can be shown that $K_{\mathrm{GaN}} \approx-1,232 \pm 205$ $\mathrm{cm}^{-1}$ for $\mathrm{GaN}$ and $K_{\mathrm{AlN}} \approx-1,472 \pm 253 \mathrm{~cm}^{-1}$ for AlN, both of which have uncertainties of almost $20 \%$.

This linear model of Raman shift (Eq. (1)) was applied to study the lateral strain distribution in GaN/AlN SL with high densities of crack networks. Figure 2(a) shows the optical microscope image of the surface of the 30-period GaN/AlN SL sample (S1) with thicknesses $t_{\mathrm{GaN}} / t_{\mathrm{AlN}} \sim 7.1 / 3.3 \mathrm{~nm}$.

We can see that the SL surface is covered with a net of microcracks (MCs) that run strictly along crystallographic directions, featuring hexagonal (six-fold) symmetry of a III-nitride wurtzite structure.

Note that on the surface, the bimodal distribution in the MC density can be observed with averaged lateral spacing between cracks about $6 \pm 3$ and $15 \pm 10 \mu \mathrm{m}$. At the same time, MCs with lateral spacing $>100 \mu \mathrm{m}$ also appear. Two different mechanisms are involved in the MCs generation that formed during the different growth stages: (i) MCs formed during the SL growth due to the large lattice mismatch, and (ii) MCs formed during cooling down processes due to the mismatch in the coefficient of thermal expansion [77]. It is obvious that cracking must lead to laterally non-uniform strain distribution in these GaN/AlN SLs. HRXRD reciprocal space maps (RSMs) measured across the asymmetric $\overline{1} 124$ and 1015 reflections were used to determine the values of the lattice parameters of the GaN and AlN SL layers averaged over a large area. On the $\overline{1} 24$ RSM in Fig. 2(b), the GaN substrate is seen as the most intense peak and the SL appears in the form of satellite peaks distributed along the $Q_{z}$ axis. Even though there is a large lattice mismatch between the GaN and AlN layers of the SL, the presence of the SL satellite peaks confirms that the coherency between those SL layers is preserved. This is true even when it is obvious that the entire SL structure is not pseudomorphic with the underlying substrate $[29,44,78]$. This means that the in-plane lattice parameter of the superlattice $\left(a_{\mathrm{SL}}\right)$ is equal to lattice parameters of the well and barrier layers, $a_{\mathrm{SL}}=a_{\mathrm{SL}}^{\mathrm{GaN}}=a_{\mathrm{SL}}^{\mathrm{AN}}$ (see Fig. $S 1$ in the ESM for details). The elongation of the satellite peaks along the $Q_{x}$ axis on the RSM is related with the varying $a_{\mathrm{SL}}$ in the SL. The vertical dashed lines mark the two prominent regions of

Table 1 Phonon deformation potentials and elastic constants for $\mathrm{GaN}$ and AlN bulks from the literature [33, 39-41, 53-76]

\begin{tabular}{ccccc}
\hline Parameters & $a_{E_{2}^{\mathrm{H}}}\left(\mathrm{cm}^{-1}\right)$ & $b_{E_{2}^{\mathrm{H}}}\left(\mathrm{cm}^{-1}\right)$ & $C_{13}(\mathrm{GPa})$ & $C_{33}(\mathrm{GPa})$ \\
\hline $\mathrm{GaN}$ & $-806 \pm 49$ & $-765 \pm 52$ & $-97 \pm 17$ & $-391 \pm 15$ \\
$\mathrm{AlN}$ & $-1,011 \pm 79$ & $-940 \pm 29$ & $-113 \pm 13$ & $-386 \pm 10$ \\
\hline
\end{tabular}

the SL peaks. An extracted cut over the satellite peak maxima on the RSM (Fig. 2(b), inset) shows that these two regions are attributed to the fully strained and partially relaxed parts of the SL. The $a_{\mathrm{SL}}$ or the lattice parameters of the GaN and AlN SL layers vary between these two extremes $\left(3.171 \AA \leq a_{\mathrm{SL}}\left(a_{\mathrm{SL}}^{\mathrm{GaN}}, a_{\mathrm{SL}}^{\mathrm{AIN}}\right) \leq\right.$ $3.186 \AA$ ), which were calculated by using the relationships

$$
\begin{aligned}
& \left\{\begin{array}{l}
\boldsymbol{Q}_{\overline{1} 115}=\sqrt{Q_{x, 1015}^{2}+Q_{z, 1015}^{2}} \\
\boldsymbol{Q}_{\overline{112} 24}=\sqrt{Q_{x, \overline{11} 24}^{2}+Q_{z, \overline{11} 24}^{2}}
\end{array}\right. \\
& Q_{x}^{2}=\frac{4}{3} \frac{h^{2}+h k+k^{2}}{a^{2}} \\
& Q_{z}^{2}=\frac{l^{2}}{c^{2}}
\end{aligned}
$$

where $a$ and $c$ are the lateral and vertical lattice parameters, $h, k$, and $l$ are Miller indices, $\boldsymbol{Q}$ is the reciprocal space vector with components $Q_{x}$ and $Q_{z}$. The strain distribution through the GaN/AlN SL affected by MCs (see Fig. 2(a)) was studied at the microscale by Raman spectroscopy. The micro-Raman spectra were measured across two MCs (Fig. 3, inset) with a step size of $\sim 2 \mu \mathrm{m}$ (the spot size of the laser light on the sample surface is $\sim 1 \mu \mathrm{m}$ ). The peaks in the Raman spectra were fitted by Gaussian line shapes. The synchronous shift of the $E_{2}^{\mathrm{H}}$ modes across the cracks is explicitly observed for both the GaN and AlN SL layers (see left frame on Fig. 3). The red shift of the $E_{2}^{\mathrm{H}}\left(\mathrm{GaN}_{\mathrm{SL}}\right)$ $\left(E_{2}^{\mathrm{H}}\left(\mathrm{AlN}_{\mathrm{SL}}\right)\right)$ is related to the increasing compressive (decreasing tensile) strain in the GaN(AlN) SL layers in the close vicinity of the crack as a result of strain relaxation in the SL. Measurements around more than ten cracks show similar values and characteristics of these SL modes. Moreover, it was observed that the strain relaxation in the SL starts approximately $15 \mu \mathrm{m}$ away from the crack, which correlates with previously reported results $[79,80]$. Raman mapping of a lateral strain distribution in $\mathrm{GaN}$ and AlN SL layers in the close vicinity of a net of MCs for sample S3 further supported this observation (see Fig. S2 in the ESM for

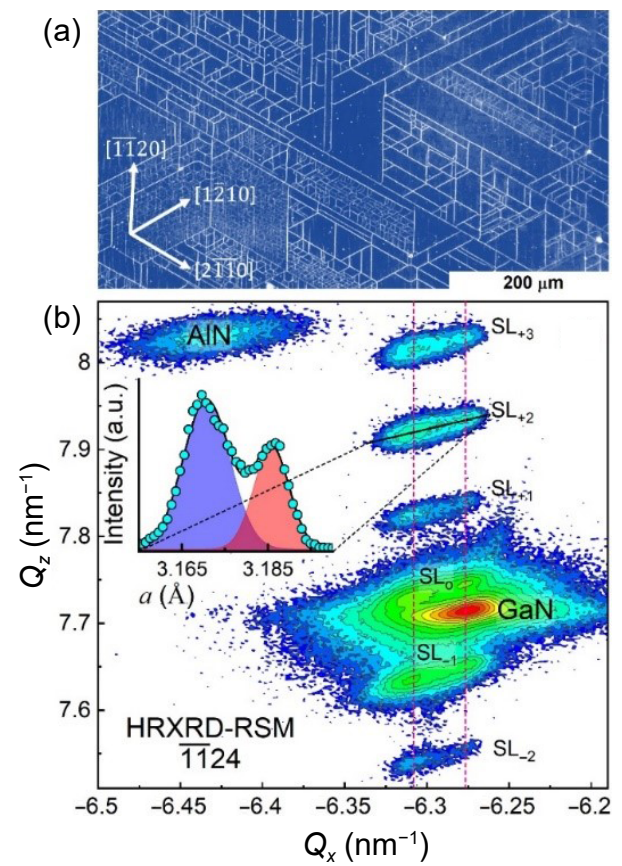

Figure 2 (a) Optical microscopic image showing a hexagonal network of cracks (white lines represent cracks propagation) on the 30-period GaN/AIN SL surface (sample S1). (b) HRXRD-RSM around the asymmetric $\overline{1} 24$ reflection for the sample S1. Inset in (b): extracted cut over the satellite peak maxima showing two distinct peaks (dots: measured intensity profile, line: Gaussian fit). 
details). Therefore, taking into account the XRD data for sample S1 (see Fig. 2(b)), it can be concluded that between cracks with a large spacing $(>\sim 30 \mu \mathrm{m})$ the SL is fully strained to the GaN substrate $\left(a_{\mathrm{SL}} \approx a_{\text {bulk }}^{\mathrm{GaN}}\right)$, whereas in the densely cracked area and in general close to a crack, the SL is partially relaxed $\left(a_{\mathrm{SL}}<a_{\mathrm{bulk}}^{\mathrm{GaN}}\right)$.

Now, the lattice parameters in the SL of the GaN and AlN would generally be calculated from the Raman frequency shift by substituting the strain in Eq. (1) with

$$
\varepsilon_{x x}=\frac{a_{\mathrm{SL}}^{\mathrm{GaN}(\mathrm{AlN})}-a_{\text {bulk }}^{\mathrm{GaN}(\mathrm{AlN})}}{a_{\mathrm{bulk}}^{\mathrm{GaN}(\mathrm{AIN})}}
$$

Then, the lattice parameters can be determined from the Raman frequency shift by

$$
a_{\mathrm{SL}}^{\mathrm{GaN}(\mathrm{AIN})}=a_{\mathrm{bulk}}^{\mathrm{GaN}(\mathrm{AlN})}\left(1+\frac{\Delta \omega_{E_{2}^{\mathrm{H}}}^{\mathrm{GaN}(\mathrm{AIN})}}{K_{\mathrm{GaN}(\mathrm{AIN})}}\right)
$$

The lattice parameters, $a_{\mathrm{SL}}^{\mathrm{GaN}}$ and $a_{\mathrm{SL}}^{\mathrm{AIN}}$, calculated according to Eq. (6) from the Raman frequency shift are plotted in the right frame of Fig. 3 with the broad bands determined by the above discussed variability in the accepted values for the deformation potentials.

These lattice parameters obviously exhibit similar synchronous changes, but they are not equal. However, as demonstrated above through the XRD RSM measurements, the coherency between the GaN and AlN SL layers is preserved, and therefore the lattice parameters of the well and barrier layers should be equal, $a_{\mathrm{SL}}=a_{\mathrm{SL}}^{\mathrm{GaN}}=a_{\mathrm{SL}}^{\mathrm{AlN}}$ (see Fig. S1 in the ESM). Moreover, as predicted by Eq. (6) the lattice parameter for the AlN SL layer is in fact much larger than even bulk GaN $\left(a_{\text {bulk }}^{\mathrm{GaN}}=3.189 \AA\right)$. This is, of course, not possible, because there is no lattice in the structure with a lattice constant larger than $\mathrm{GaN}$, which could strain the AlN $\left(a_{\text {bulk }}^{\text {AlN }}=3.113 \AA\right)$ to this large size. In other words, in the GaN/AlN SL fully strained to the GaN substrate, the maximum value of the lattice parameter for the AlN SL layer should only be $a_{\mathrm{SL}}^{\mathrm{AIN}}=a_{\mathrm{SL}}^{\mathrm{GaN}}=a_{\mathrm{bulk}}^{\mathrm{GaN}}$. Furthermore, the deviation of the Raman derived value of the AlN lattice parameter from SL lattice parameter derived from the XRD is much larger than the Raman derived value of the GaN lattice parameter from the SL lattice parameter derived from the $\mathrm{XRD}$. This is believed to be due to the much larger amount of strain stored in the AlN layer. As can be seen on Fig. 3 (right frame), the values of $a_{\mathrm{SL}}^{\mathrm{GaN}}$ calculated from the Raman shift are almost within the range of $a_{\mathrm{SL}}$ determined from the XRD measurements. Therefore, the Raman shift, if using Eqs.
(1) and (6), must not provide the correct values of strain and lattice parameters, respectively, not only for high-strained AlN, but also for the less-strained GaN.

The linear model in Eq. (1) was also not able to explain the observed Raman shifts for highly strained GaN QWs embedded in an AlN matrix [33]. This experimental data from Ref. [33] as well as the phonon frequency strain relation for GaN using linear model (Eq. (1)) are shown in Fig. 4(a) as the star symbols and gray shaded area, respectively. The relation of $E_{2}^{\mathrm{H}}$ vs. $\varepsilon_{x x}$ for AlN is shown in Fig. 4(b). The data $E_{2}^{\mathrm{H}}$ vs. $\varepsilon_{x x}$ taken near a crack for sample S1 correlate with previously reported results, i.e. the Raman shift for the relatively less strained GaN QWs is closer to the value predicted by the linear model (Eq. (1)) than the Raman shift for the highly strained AlN barriers.

In the same work [33] it was concluded that the Raman shift for the highly strained GaN QWs $\left(2.2 \%<\varepsilon_{x x}<2.5 \%\right)$ falls out of the prediction of the linear model that was proved on the experimental data limited to a small biaxial strain $\left(\varepsilon_{x x}<0.2 \%\right)$. Therefore, in order to obtain the Raman shift for GaN and AlN with values of strain between small and large, a series of 30-period GaN/AlN SLs with different thickness ratios $t_{\mathrm{GaN}} / t_{\mathrm{AlN}}$ were grown on GaN-on-sapphire substrate. The measured Raman shift, and the SL period, thickness, and strain state of a quantum well/barrier of GaN/AlN SLs extracted by HRXRD-RSM according to the technique proposed in Ref. [29] are shown in Table 2. The measured values of $E_{2}^{\mathrm{H}}$ vs. $\varepsilon_{x x}$ taken near a crack are plotted in Fig. 4(a) for GaN and in Fig. 4(b) for AlN. The $E_{2}^{\mathrm{H}}\left(\mathrm{GaN}_{\text {substr. }}\right)$ and $E_{2}^{\mathrm{H}}\left(\mathrm{AlN}_{\text {substr. }}\right)$ Raman shifts for $\mathrm{GaN}$ and $\mathrm{AlN}$ substrate layers are shown at small strain values. The deviation of the measured Raman shift from the linear model (Eq. (1)) increases with increasing strain for both GaN and AlN SL layers. In order to describe this new experimental dependence of the Raman frequency vs. biaxial strain, a simple mass-spring harmonic oscillator model was proposed [33]. In the equation for the oscillation frequency $\omega=\sqrt{k / m}$, the force constant $k$ can be considered as the bond strength between atoms with the mass $m$. The constant $k$ is inversely proportional to the lattice constant (bond length) and the $k$ vs. strain $\left(\varepsilon_{x x}\right)$ relation can be modeled by $k\left(\varepsilon_{x x}\right)=k_{\mathrm{o}}\left(1-R \varepsilon_{x x}\right)$, where $k_{\mathrm{o}}=\omega_{\mathrm{o}}{ }^{2} / m$ is the bond strenght of unstrained material and $R$ is a constant. Therefore, the Raman phonon frequency $(\omega)$ vs. strain $\left(\varepsilon_{x x}\right)$ in this simple model is

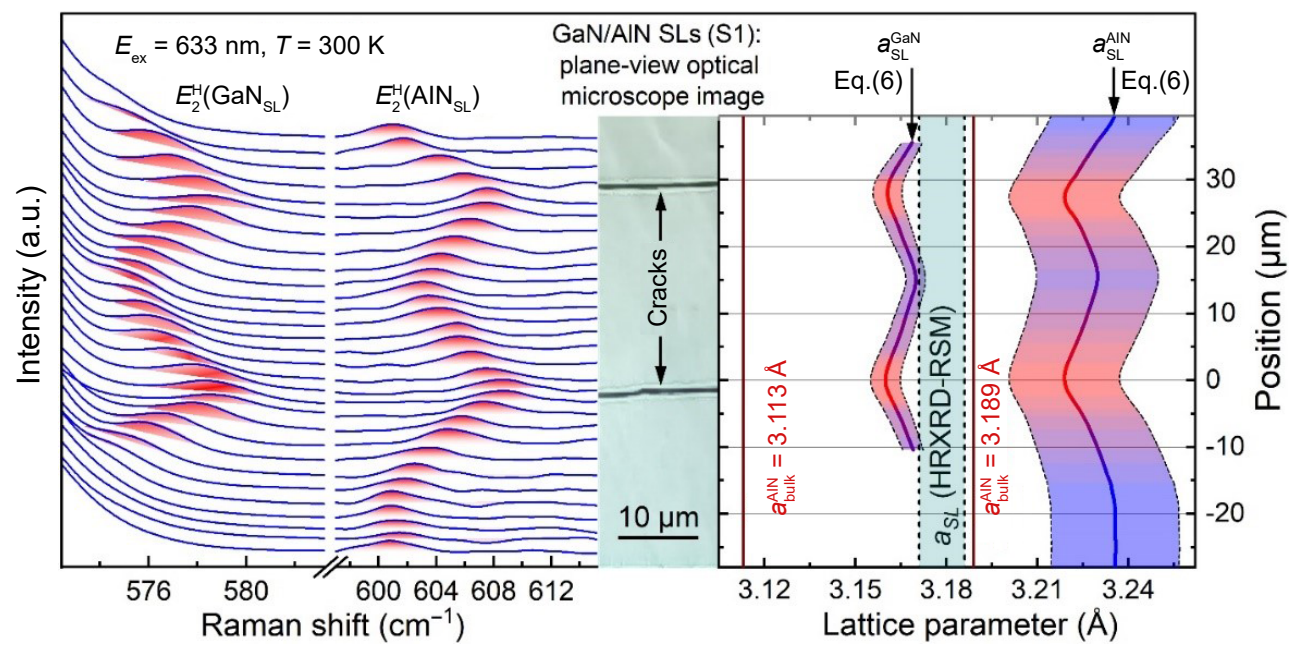

Figure 3 Micro-Raman spectra (left frame) measured across two cracks for GaN/AlN SL (Fig. 2, sample S1). Optical microscope image showing the two obvious cracks across which the Raman spectra on the left were measured. In the right frame: (i) curved lines show the average (solid) and error range (dashed) of the calculated lattice parameters $a_{\mathrm{SL}}^{\mathrm{GaN}}$ and $a_{\mathrm{SL}}^{\mathrm{AlN}}$ using Eq. (6) of phonon frequency shift vs. lattice parameter; (ii) vertical solid lines show the lattice parameters of the bulk GaN and AlN; (iii) vertical dashed lines show the range of $a_{\mathrm{SL}}$ or the lattice parameters of the GaN and AlN SL layers extracted from HRXRD-RSM data (see Fig. 2(b)). 
(a)

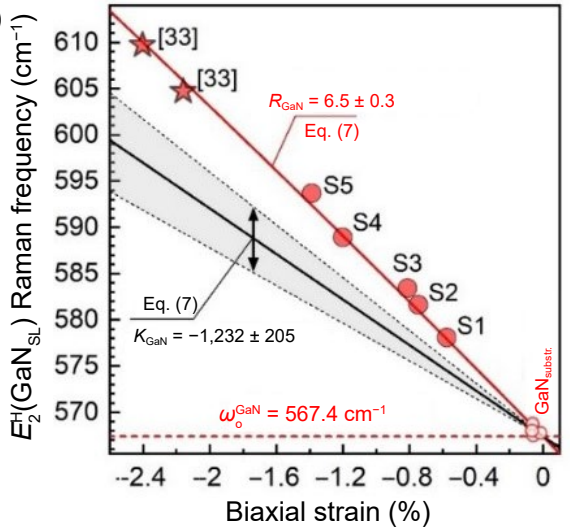

(b)

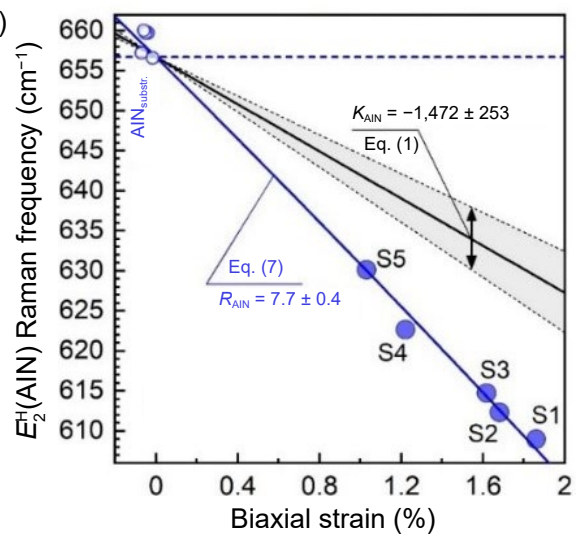

Figure 4 Raman peak frequency of the $E_{2}^{\mathrm{H}}$ phonon mode vs. the biaxial strain $\varepsilon_{x x}$ for GaN (a) and AlN (b). The gray shaded regions show the relation of $E_{2}^{\mathrm{H}}$ vs. $\varepsilon_{x x}$ using the linear model (Eq. (1)) along with the range of $K$ values from the literature. The blue lines show the relation of $E_{2}^{\mathrm{H}}$ vs. $\varepsilon_{x x}$ using the nonlinear model (Eq. (7)). The symbols show the experimental data for GaN/AlN SLs presented in Table 2 (circles) and for GaN QWs embedded in an AlN matrix (stars) from Ref. [33].

$$
\omega\left(\varepsilon_{x x}\right)=\sqrt{\frac{k}{m}}=\sqrt{\frac{k_{0}\left(1-R \varepsilon_{x x}\right)}{m}}=\omega_{0} \sqrt{\left(1-R \varepsilon_{x x}\right)}
$$

A good fit of the experimental data as shown as the red and blue curves in Fig. 4 was obtained for GaN with $R_{\mathrm{GaN}}=6.5 \pm 0.3$ and for AlN with $R_{\mathrm{AlN}}=7.7 \pm 0.4$, respectively. In order to validate the new proposed approach of $E_{2}^{\mathrm{H}}$ vs. $\varepsilon_{x x}$ and determine the constants, $R_{\mathrm{GaN}(\mathrm{AlN})}$, the lattice parameters from the GaN/AIN SL are determined from the Raman data by substituting the strain from Eq. (5), in Eq. (7). Then we have

$$
a_{\mathrm{SL}}^{\mathrm{GaN}(\mathrm{AIN})}=a_{\mathrm{bulk}}^{\mathrm{GaN}(\mathrm{AlN})}\left(1+\frac{1-\left(\omega_{\mathrm{SL}}^{\mathrm{GaN}(\mathrm{AlN})} / \omega_{\mathrm{o}}^{\mathrm{GaN}(\mathrm{AIN})}\right)^{2}}{R_{\mathrm{GaN}(\mathrm{AlN})}}\right)
$$

This results in the fitting parameters, $R_{\mathrm{GaN}}=6.5$ and $R_{\mathrm{AlN}}=7.7$. The resulting relations are plotted in Fig. 5 as a dashed line for GaN and AlN along with their respective experimental data from samples S1-S5 as symbols in addition to many examples from the literature.

As can be seen, throughout the entire range between the $\mathrm{GaN}$ and AlN bulk parameters, there is a good fit. This means that Eq. (8) provides the correct lattice parameters for SL layers, i.e., $a_{\mathrm{SL}}=a_{\mathrm{SL}}^{\mathrm{GaN}}=a_{\mathrm{SL}}^{\mathrm{AN}}$. Therefore, it can be concluded that the new approach of $E_{2}^{\mathrm{H}}$ vs. $\varepsilon_{x x}$ using Eq. (7) with $R_{\mathrm{GaN}}=6.5 \pm 0.3$ and $R_{\mathrm{AIN}}$ $=7.7 \pm 0.4$ can be used to correctly estimate the biaxial strain in IIInitrides via Raman spectroscopy. The considerable application potential of the present findings could benefit a broad readership interested in III-nitride-based optoelectronics and power electronics devices [1-20, 85-88].

\section{Conclusions}

Strain-induced polarization is one of the key parameters for tuning the optical and electrical properties of III-nitrides. At the same time, Raman spectroscopy is a widely used method for rapid measurement of strain in nano- and hetero-structures. Previously, Raman measurements of strain in III-nitrides have been based on a linear model with experiments limited to a small range of biaxial strains $\left(0 \%<\varepsilon_{x x}<0.2 \%\right)[34,40]$. Here, for a series of 30 -period GaN/AlN SLs with different thickness ratios $\left(t_{\mathrm{GaN}} / t_{\mathrm{AlN}}\right)$, and consequently with different residual strains, we found a large discrepancy between the experimental values of strain measured by HRXRD and that calculated via Raman spectroscopy by using the standard model. Moreover, we have shown that this discrepancy increases as the strain in both the GaN and AlN layers increases. We propose a new model that describes the straininduced Raman frequency shift in GaN and AlN for a wide range of biaxial strains (up to 2.5\%). Finally, we proposed a new approach to correlate the Raman frequency shift and strain, which is based on the lattice coherency in the epitaxial layers of SL

\begin{tabular}{|c|c|c|c|c|c|c|}
\hline & \multicolumn{6}{|c|}{ Raman data } \\
\hline & \multicolumn{2}{|c|}{$\omega_{E_{2}^{\mathrm{H}}}^{\mathrm{GaN}_{\text {substr. }}}\left(\mathrm{cm}^{-1}\right)$} & \multicolumn{2}{|l|}{$\omega_{E_{2}^{\mathrm{H}}}^{\mathrm{AlN}} \mathrm{Aubstr}\left(\mathrm{cm}^{-1}\right)$} & $\omega_{E_{2}^{\mathrm{H}}}^{\mathrm{GaN}_{\mathrm{SL}}}\left(\mathrm{cm}^{-1}\right)$ & $\omega_{E_{2}^{\mathrm{H}}}^{\mathrm{AlN}}\left(\mathrm{cm}^{-1}\right)$ \\
\hline $\mathrm{S} 1$ & \multicolumn{2}{|c|}{$568.54 \pm 0.30$} & \multicolumn{2}{|l|}{$657.26 \pm 0.57$} & $578.10 \pm 1.15$ & $609.01 \pm 0.62$ \\
\hline $\mathrm{S} 2$ & \multicolumn{2}{|c|}{$567.51 \pm 0.11$} & \multicolumn{2}{|l|}{$659.78 \pm 0.16$} & $581.65 \pm 0.36$ & $612.38 \pm 0.70$ \\
\hline S3 & \multicolumn{2}{|c|}{$568.79 \pm 0.08$} & \multicolumn{2}{|l|}{$656.65 \pm 0.47$} & $583.45 \pm 0.94$ & $614.79 \pm 1.38$ \\
\hline S4 & \multicolumn{2}{|c|}{$567.76 \pm 0.15$} & \multicolumn{2}{|l|}{$659.78 \pm 0.18$} & $588.95 \pm 0.28$ & $622.72 \pm 0.69$ \\
\hline \multirow[t]{3}{*}{ S5 } & \multicolumn{2}{|c|}{$568.03 \pm 0.09$} & \multicolumn{2}{|l|}{$660.05 \pm 0.24$} & $593.73 \pm 2.00$ & $630.18 \pm 1.75$ \\
\hline & \multicolumn{6}{|c|}{ HRXRD-RSM data } \\
\hline & $\varepsilon_{x x}^{\mathrm{GaN}_{\text {substr. }}\left(\times 10^{-4}\right)}$ & $\varepsilon_{x x}^{\mathrm{AlN}_{\text {substr. }}\left(\times 10^{-4}\right)}$ & $T_{\mathrm{SL}}(\mathrm{nm})$ & $t_{\mathrm{GaN}} / t_{\mathrm{AlN}}(\mathrm{nm})$ & $\varepsilon_{x x}^{\mathrm{GaN}_{\mathrm{SL}}}\left(\times 10^{-2}\right)$ & $\varepsilon_{x x}^{\mathrm{AlN}_{\mathrm{SL}}}\left(\times 10^{-2}\right)$ \\
\hline S1 & -6.17 & -7.04 & $10.47 \pm 0.6$ & $7.13 / 3.34$ & -0.616 & 1.810 \\
\hline S2 & -5.98 & -4.67 & $8.92 \pm 0.03$ & $5.51 / 3.41$ & -0.821 & 1.601 \\
\hline S3 & -6.38 & -1.60 & $7.95 \pm 0.08$ & $4.61 / 3.33$ & -0.924 & 1.494 \\
\hline S4 & -1.65 & -4.13 & $6.35 \pm 0.04$ & $2.83 / 3.52$ & -1.241 & 1.170 \\
\hline S5 & -6.67 & -6.00 & $4.84 \pm 0.08$ & $1.38 / 3.47$ & -1.516 & 0.888 \\
\hline
\end{tabular}

Table 2 Raman frequency $\left(E_{2}^{\mathrm{H}}\right)$, in-plane strain and thickness for GaN and AlN layers in GaN/AlN SLs calculated from HRXRD-RSM [29] 


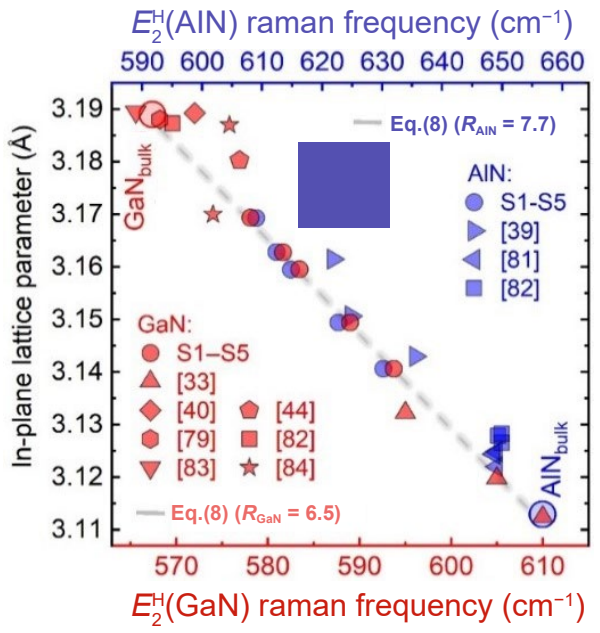

Figure 5 The in-plane lattice parameter vs. Raman frequency $E_{2}^{\mathrm{H}}$ calculated according to Eq. (8) (dashed line) for GaN with $R_{\mathrm{GaN}}=6.5$ and for AlN with $R_{\mathrm{AIN}}=7.7$. The red and blue symbols represent the experimental data for GaN and AlN for samples S1-S5 (Table 1) as well as from the literature.

structures and should be able to be used for a wide range of materials. The results offer a fast, nondestructive, and precise method for measuring strain in III-nitrides from GaN to AlN and can be readily applied to other semiconductor materials.

\section{Acknowledgements}

This work was supported by the U.S. National Science Foundation Engineering Research Center for Power Optimization of Electro Thermal Systems (POETS) with cooperative agreement EEC1449548. F. M. O. and M. D. T. acknowledge the financial support of Coordenação de Aperfeiçoamento de Pessoal de Nível Superior - Brasil (CAPES) - Finance Code 001.

Electronic Supplementary Material: Supplementary material (Table S1: Values of bulk phonon deformation potentials and elastic constants for GaN and AlN from each reference used in Table 1, Fig. S1: Lattice parameters of SL layers using Eq. (8), and Fig. S2: Raman mapping using Eq. (7)) is available in the online version of this article at https://doi.org/10.1007/s12274-0213855-4.

\section{References}

[1] Wu, W. Z.; Wang, Z. L. Piezotronics and piezo-phototronics for adaptive electronics and optoelectronics. Nat. Rev. Mater. 2016, 1 , 16031.

[2] Wang, D. H.; Liu, X.; Fang, S.; Huang, C.; Kang, Y.; Yu, H. B.; Liu, Z. L.; Zhang, H. C.; Long, R.; Xiong, Y. J. et al. Pt/AlGaN nanoarchitecture: Toward high responsivity, self-powered ultravioletsensitive photodetection. Nano Lett. 2021, 21, 120-129.

[3] Bishop, S. G.; Hadden, J. P.; Alzahrani, F. D.; Hekmati, R.; Huffaker, D. L.; Langbein, W. W.; Bennett, A. J. Room-temperature quantum emitter in aluminum nitride. ACS Photonics 2020, 7, $1636-1641$.

[4] Tamariz, S.; Callsen, G.; Stachurski, J.; Shojiki, K.; Butté, R.; Grandjean, N. Toward bright and pure single photon emitters at 300 $\mathrm{K}$ based on GaN quantum dots on silicon. ACS Photonics 2020, 7, $1515-1522$.

[5] Arita, M.; Le Roux, F.; Holmes, M. J.; Kako, S.; Arakawa, Y. Ultraclean single photon emission from a GaN quantum dot. Nano Lett. 2017, 17, 2902-2907.

[6] Zhou, Y.; Wang, Z. Y.; Rasmita, A.; Kim, S.; Berhane, A.; Bodrog, Z.; Adamo, G.; Gali, A.; Aharonovich, I.; Gao, W. B. Room temperature solid-state quantum emitters in the telecom range. Sci. $A d v . \mathbf{2 0 1 8}$, 4, eaar3580.
[7] Growden, T. A.; Storm, D. F.; Cornuelle, E. M.; Brown, E. R.; Zhang, W. D.; Downey, B. P.; Roussos, J. A.; Cronk, N.; Ruppalt, L. B.; Champlain, J. G. et al. Superior growth, yield, repeatability, and switching performance in GaN-based resonant tunneling diodes. Appl. Phys. Lett. 2020, 116, 113501.

[8] Qi, M.; Li, G. W.; Ganguly, S.; Zhao, P.; Yan, X. D.; Verma, J.; Song, B.; Zhu, M. D.; Nomoto, K.; Xing, H. L. et al. Strained GaN quantum-well FETs on single crystal bulk AlN substrates. Appl. Phys. Lett. 2017, 110, 063501.

[9] Diez, S.; Mohanty, S.; Kurdak, C.; Ahmadi, E. Record high electron mobility and low sheet resistance on scaled-channel N-polar GaN/AIN heterostructures grown on on-axis N-polar GaN substrates by plasma-assisted molecular beam epitaxy. Appl. Phys. Lett. 2020, 117, 042102 .

[10] Chen, H. D.; Wang, P.; Wang, X. Y.; Wang, X. F.; Rao, L. J.; Qian, Y. P.; Yin, H. J.; Hou, X. H.; Ye, H. P.; Zhou, G. F. et al. 3D InGaN nanowire arrays on oblique pyramid-textured $\mathrm{Si}$ (311) for light trapping and solar water splitting enhancement. Nano Energy 2021, 83,105768

[11] Wang, P.; Chen, H. D.; Wang, H.; Wang, X. Y.; Yin, H. J.; Rao, L. J.; Zhou, G. F.; Nötzel, R. Multi-wavelength light emission from InGaN nanowires on pyramid-textured $\mathrm{Si}(100)$ substrate grown by stationary plasma-assisted molecular beam epitaxy. Nanoscale 2020, 12, 8836-8846.

[12] Wang, P.; Chen, H. D.; Wang, H.; Wang, D.; Song, C. K.; Wang, X. Y.; Yin, H. J.; Rao, L. J.; Zhou, G. F.; Nötzel, R. Inter-facet composition modulation of III-nitride nanowires over pyramid textured Si substrates by stationary molecular beam epitaxy. Nano Res. 2021, 14, 1502-1511.

[13] Siladie, A. M.; Jacopin, G.; Cros, A.; Garro, N.; Robin, E.; Caliste, D.; Pochet, P.; Donatini, F.; Pernot, J.; Daudin, B. $\mathrm{Mg}$ and In codoped p-type AlN nanowires for pn junction realization. Nano Lett. 2019, 19, 8357-8364.

[14] Xu, H. Q.; Jiang, J. A.; Dai, Y. J.; Cui, M.; Li, K. H.; Ge, X. T.; Hoo, J.; Yan, L.; Guo, S. P.; Ning, J. Q. et al. Polarity control and nanoscale optical characterization of AlGaN-based multiplequantum-wells for ultraviolet C emitters. ACS Appl. Nano Mater. 2020, 3, 5335-5342.

[15] Lobo-Ploch, N.; Mehnke, F.; Sulmoni, L.; Cho, H. K.; Guttmann, M.; Glaab, J.; Hilbrich, K.; Wernicke, T.; Einfeldt, S.; Kneissl, M. Milliwatt power $233 \mathrm{~nm}$ AlGaN-based deep UV-LEDs on sapphire substrates. Appl. Phys. Lett. 2020, 117, 111102.

[16] Grenier, V.; Finot, S.; Jacopin, G.; Bougerol, C.; Robin, E.; Mollard, N.; Gayral, B.; Monroy, E.; Eymery, J.; Durand, C. UV emission from $\mathrm{GaN}$ wires with m-plane core-shell $\mathrm{GaN} / \mathrm{AlGaN}$ multiple quantum wells. ACS Appl. Mater. Interfaces 2020, 12, 44007-44016.

[17] Knauer, A.; Kolbe, T.; Rass, J.; Cho, H. K.; Netzel, C.; Hagedorn, S.; Lobo-Ploch, N.; Ruschel, J.; Glaab, J.; Einfeldt, S. et al. High power UVB light emitting diodes with optimized n-AlGaN contact layers. Jpn. J. Appl. Phys. 2019, 58, SCCC02.

[18] Pandey, A.; Shin, W. J.; Gim, J.; Hovden, R.; Mi, Z. High-efficiency AlGaN/GaN/AlGaN tunnel junction ultraviolet light-emitting diodes. Photon. Res. 2020, 8, 331-337.

[19] Inagaki, H.; Saito, A.; Sugiyama, H.; Okabayashi, T.; Fujimoto, S. Rapid inactivation of SARS-CoV-2 with deep-UV LED irradiation. Emerging Microbes Infect. 2020, 9, 1744-1747.

[20] Raeiszadeh, M.; Adeli, B. A Critical review on ultraviolet disinfection systems against COVID-19 outbreak: Applicability, validation, and safety considerations. ACS Photonics 2020, 7, 2941-2951.

[21] Schlichting, S.; Hönig, G. M. O.; Müßener, J.; Hille, P.; Grieb, T.; Westerkamp, S.; Teubert, J.; Schörmann, J.; Wagner, M. R.; Rosenauer, A. et al. Suppression of the quantum-confined Stark effect in polar nitride heterostructures. Commun. Phys. 2018, 1, 48.

[22] Guo, W.; Sun, H. D.; Torre, B.; Li, J. M.; Sheikhi, M.; Jiang, J. A.; Li, H. W.; Guo, S. P.; Li, K. H.; Lin, R. H. et al. Lateral-polarity structure of AlGaN quantum wells: A promising approach to enhancing the ultraviolet luminescence. Adv. Funct. Mater. 2018, 28, 1802395.

[23] Simon, J.; Protasenko, V.; Lian, C. X.; Xing, H. L.; Jena, D. 
Polarization-induced hole doping in wide-band-gap uniaxial semiconductor heterostructures. Science 2010, 327, 60-64.

[24] May, B. J.; Belz, M. R.; Ahamed, A.; Sarwar, A. T. M. G.; Selcu, C. M.; Myers, R. C. Nanoscale electronic conditioning for improvement of nanowire light-emitting-diode efficiency. ACS Nano 2018, 12, 3551-3556.

[25] Kuchuk, A. V.; Lytvyn, P. M.; Li, C.; Stanchu, H. V.; Mazur, Y. I.; Ware, M. E.; Benamara, M.; Ratajczak, R.; Dorogan, V.; Kladko, V. P. et al. Nanoscale electrostructural characterization of compositionally graded $\mathrm{Al}_{X} \mathrm{Ga}_{1-X} \mathrm{~N}$ heterostructures on GaN/Sapphire (0001) substrate. ACS Appl. Mater. Interfaces 2015, 7, 23320-23327.

[26] Li, S. B.; Ware, M.; Wu, J.; Minor, P.; Wang, Z. M.; Wu, Z. M.; Jiang, Y. D.; Salamo, G. J. Polarization induced pn-junction without dopant in graded AlGaN coherently strained on GaN. Appl. Phys. Lett. 2012, 101, 122103.

[27] Lytvyn, P. M.; Kuchuk, A. V.; Mazur, Y. I.; Li, C.; Ware, M. E.; Wang, Z. M.; Kladko, V. P.; Belyaev, A. E.; Salamo, G. J. Polarization effects in graded $\mathrm{AlGaN}$ nanolayers revealed by currentsensing and kelvin probe microscopy. ACS Appl. Mater. Interfaces 2018, 10, 6755-6763.

[28] Stanchu, H.; Der Maur, M. A.; Kuchuk, A. V.; Mazur, Y. I.; Sobanska, M.; Zytkiewicz, Z. R.; Wu, S.; Wang, Z.; Salamo, G. Compositionally graded AlGaN nanostructures: Strain distribution and X-ray diffraction reciprocal space mapping. Cryst. Growth Des. 2020, 20, 1543-1551.

[29] Stanchu, H. V.; Kuchuk, A. V.; Barchuk, M.; Mazur, Y. I.; Kladko, V. P.; Wang, Z. M.; Rafaja, D.; Salamo, G. J. Asymmetrical reciprocal space mapping using X-ray diffraction: A technique for structural characterization of GaN/AlN superlattices. CrystEngComm 2017, 19, 2977-2982.

[30] Kuchuk, A. V.; Stanchu, H. V.; Li, C.; Ware, M. E.; Mazur, Y. I.; Kladko, V. P.; Belyaev, A. E.; Salamo, G. J. Measuring the depth profiles of strain/composition in AlGaN-graded layer by highresolution X-ray diffraction. J. Appl. Phys. 2014, 116, 224302.

[31] Hetzl, M.; Kraut, M.; Winnerl, J.; Francaviglia, L.; Doblinger, M.; Matich, S.; Morral, A. F. I.; Stutzmann, M. Strain-induced band gap engineering in selectively grown $\mathrm{GaN}-(\mathrm{Al}, \mathrm{Ga}) \mathrm{N}$ core-shell nanowire heterostructures. Nano Lett. 2016, 16, 7098-7106.

[32] Okamoto, S.; Saito, N.; Ito, K.; Ma, B.; Morita, K.; Iida, D.; Ohkawa, K.; Ishitani, Y. Energy transport analysis in a $\mathrm{Ga}_{0.84} \mathrm{In}_{0.16} \mathrm{~N} / \mathrm{GaN}$ heterostructure using microscopic Raman images employing simultaneous coaxial irradiation of two lasers. Appl. Phys. Lett. 2020, 116, 142107.

[33] Qi, M.; Li, G. W.; Protasenko, V.; Zhao, P.; Verma, J.; Song, B.; Ganguly, S.; Zhu, M. D.; Hu, Z. Y.; Yan, X. D. et al. Dual optical marker Raman characterization of strained GaN-channels on AlN using AlN/GaN/AlN quantum wells and ${ }^{15} \mathrm{~N}$ isotopes. Appl. Phys. Lett. 2015, 106, 041906.

[34] Liu, E.; Conti, F.; Bhogaraju, S. K.; Signorini, R.; Pedron, D.; Wunderle, B.; Elger, G. Thermomechanical stress in GaN-LEDs soldered onto $\mathrm{Cu}$ substrates studied using finite element method and Raman spectroscopy. J. Raman Spectrosc. 2020, 51, 2083-2094.

[35] Ma, B.; Tang, M. C.; Ueno, K.; Kobayashi, A.; Morita, K.; Fujioka, H.; Ishitani, Y. Combined infrared reflectance and Raman spectroscopy analysis of Si-doping limit of GaN. Appl. Phys. Lett. 2020, 117, 192103.

[36] Hammarberg, S.; Dagyte, V.; Chayanun, L.; Hill, M. O.; Wyke, A.; Björling, A.; Johansson, U.; Kalbfleisch, S.; Heurlin, M.; Lauhon, L. $\mathrm{J}$. et al. High resolution strain mapping of a single axially heterostructured nanowire using scanning X-ray diffraction. Nano Res. 2020, 13, 2460-2468.

[37] Sarkar, K.; Datta, D.; Gosztola, D. J.; Shi, F. Y.; Nicholls, A.; Stroscio, M. A.; Dutta, M. Raman analysis of phonon modes in a short period AlN/GaN superlattice. Superlattices Microstruct. 2018, $115,116-122$

[38] Wallentin, J.; Jacobsson, D.; Osterhoff, M.; Borgström, M. T.; Salditt, T. Bending and twisting lattice tilt in strained core-shell nanowires revealed by nanofocused X-ray diffraction. Nano Lett. 2017, 17, 4143-4150.

[39] Kolomys, O.; Tsykaniuk, B.; Strelchuk, V.; Naumov, A.; Kladko,
V.; Mazur, Y. I.; Ware, M. E.; Li, S. B.; Kuchuk, A.; Maidaniuk, Y. et al. Optical and structural study of deformation states in the GaN/AlN superlattices. J. Appl. Phys. 2017, 122, 155302.

[40] Davydov, V. Y.; Averkiev, N. S.; Goncharuk, I. N.; Nelson, D. K.; Nikitina, I. P.; Polkovnikov, A. S.; Smirnov, A. N.; Jacobson, M. A.; Semchinova, O. K. Raman and photoluminescence studies of biaxial strain in GaN epitaxial layers grown on 6H-SiC. J. Appl. Phys. 1997, $82,5097-5102$

[41] Davydov, V. Y.; Kitaev, Y. E.; Goncharuk, I. N.; Smirnov, A. N.; Graul, J.; Semchinova, O.; Uffmann, D.; Smirnov, M. B.; Mirgorodsky, A. P.; Evarestov, R. A. Phonon dispersion and Raman scattering in hexagonal GaN and AlN. Phys. Rev. B 1998, 58, 12899-12907.

[42] Zhang, L. Full optical phonon states and their dispersive spectra of a wurtzite GaN/AlGaN superlattice: Quantum size effect. Phys. Status Solidi B 2011, 248, 2120-2127.

[43] Park, K.; Mohamed, A.; Dutta, M.; Stroscio, M. A.; Bayram, C. Electron scattering via interface optical phonons with high group velocity in wurtzite GaN-based quantum well heterostructure. Sci. Rep. 2018, 8, 15947.

[44] Darakchieva, V.; Valcheva, E.; Paskov, P. P.; Schubert, M.; Paskova, T.; Monemar, B.; Amano, H.; Akasaki, I. Phonon mode behavior in strained wurtzite AlN/GaN superlattices. Phys. Rev. B 2005, 71, 115329 .

[45] Manjón, F. J.; Errandonea, D.; Romero, A. H.; Garro, N.; Serrano, J.; Kuball, M. Lattice dynamics of wurtzite and rocksalt AlN under high pressure: Effect of compression on the crystal anisotropy of wurtzitetype semiconductors. Phys. Rev. B 2008, 77, 205204.

[46] Sanjurjo, J. A.; López-Cruz, E.; Vogl, P.; Cardona, M. Dependence on volume of the phonon frequencies and the ir effective charges of several III-V semiconductors. Phys. Rev. B 1983, 28, 4579-4584.

[47] Kuball, M.; Hayes, J. M.; Prins, A. D.; Van Uden, N. W. A.; Dunstan, D. J.; Shi, Y.; Edgar, J. H. Raman scattering studies on single-crystalline bulk AlN under high pressures. Appl. Phys. Lett. 2001, 78, 724-726.

[48] Goñi, A. R.; Siegle, H.; Syassen, K.; Thomsen, C.; Wagner, J. M. Effect of pressure on optical phonon modes and transverse effective charges in GaN and AlN. Phys. Rev. B 2001, 64, 035205.

[49] Yakovenko, E. V.; Gauthier, M.; Polian, A. High-pressure behavior of the bond-bending mode of AIN. J. Exp. Theor. Phys. 2004, 98, 981-985.

[50] Perlin, P.; Polian, A.; Suski, T. Raman-scattering studies of aluminum nitride at high pressure. Phys. Rev. B 1993, 47, 2874-2877.

[51] Hibberd, M. T.; Frey, V.; Spencer, B. F.; Mitchell, P. W.; Dawson, P.; Kappers, M. J.; Oliver, R. A.; Humphreys, C. J.; Graham, D. M. Dielectric response of wurtzite gallium nitride in the terahertz frequency range. Solid State Commun. 2016, 247, 68-71.

[52] Levinshtein, M. E.; Rumyantsev, S. L.; Shur, M. S. Properties of Advanced Semiconductor Materials: GaN, AIN, InN, BN, SiC, SiGe; Wiley: New York, 2001.

[53] Yang, S. B.; Miyagawa, R.; Miyake, H.; Hiramatsu, K.; Harima, H. Raman scattering spectroscopy of residual stresses in epitaxial AlN films. Appl. Phys. Express 2011, 4, 031001.

[54] Demangeot, F.; Frandon, J.; Baules, P.; Natali, F.; Semond, F.; Massies, J. Phonon deformation potentials in hexagonal GaN. Phys. Rev. B 2004, 69, 155215

[55] Davydov, S. Y. Evaluation of physical parameters for the group III nitrates: BN, AlN, GaN, and InN. Semiconductors 2002, 36, 41-44.

[56] Ambacher, O.; Majewski, J.; Miskys, C.; Link, A.; Hermann, M.; Eickhoff, M.; Stutzmann, M.; Bernardini, F.; Fiorentini, V.; Tilak, V. et al. Pyroelectric properties of $\mathrm{Al}(\mathrm{In}) \mathrm{GaN} / \mathrm{GaN}$ hetero- and quantum well structures. J. Phys. :Condens. Matter 2002, 14, 3399-3434.

[57] Wagner, J. M.; Bechstedt, F. Properties of strained wurtzite GaN and AlN: Ab initio studies. Phys. Rev. B 2002, 66, 115202.

[58] Sarua, A.; Kuball, M.; Van Nostrand, J. E. Deformation potentials of the $E_{2}$ (high) phonon mode of AlN. Appl. Phys. Lett. 2002, 81, 1426-1428.

[59] Reeber, R. R.; Wang, K. High temperature elastic constant prediction of some group III-nitrides. MRS Internet J. Nitride Semicond. Res. 2001, 6,3 . 
[60] Wagner, J. M.; Bechstedt, F. Phonon deformation potentials of $\alpha$ GaN and -AlN: An ab initio calculation. Appl. Phys. Lett. 2000, 77, 346-348.

[61] Nowak, R.; Pessa, M.; Suganuma, M.; Leszczynski, M.; Grzegory, I.; Porowski, S.; Yoshida, F. Elastic and plastic properties of GaN determined by nano-indentation of bulk crystal. Appl. Phys. Lett. 1999, 75, 2070-2072.

[62] Deguchi, T.; Ichiryu, D.; Toshikawa, K.; Sekiguchi, K.; Sota, T.; Matsuo, R.; Azuhata, T.; Yamaguchi, M.; Yagi, T.; Chichibu, S. et al. Structural and vibrational properties of GaN. J. Appl. Phys. 1999, 86, 1860-1866.

[63] Gleize, J.; Demangeot, F.; Frandon, J.; Renucci, M. A.; Widmann, F.; Daudin, B. Phonons in a strained hexagonal GaN-AlN superlattice. Appl. Phys. Lett. 1999, 74, 703-705.

[64] Shimada, K.; Sota, T.; Suzuki, K. First-principles study on electronic and elastic properties of BN, AlN, and GaN. J. Appl. Phys. 1998, 84, 4951-4958.

[65] Deger, C.; Born, E.; Angerer, H.; Ambacher, O.; Stutzmann, M.; Hornsteiner, J.; Riha, E.; Fischerauer, G. Sound velocity of $\mathrm{Al}_{x} \mathrm{Ga}_{1-x} \mathrm{~N}$ thin films obtained by surface acoustic-wave measurements. Appl. Phys. Lett. 1998, 72, 2400-2402.

[66] Kim, K.; Lambrecht, W. R. L.; Segall, B. Elastic constants and related properties of tetrahedrally bonded $\mathrm{BN}, \mathrm{AlN}, \mathrm{GaN}$, and $\mathrm{InN}$. Phys. Rev. B 1996, 53, 16310-16326.

[67] Wright, A. F. Elastic properties of zinc-blende and wurtzite AlN, GaN, and InN. J. Appl. Phys. 1997, 82, 2833-2839.

[68] Yamaguchi, M.; Yagi, T.; Azuhata, T.; Sota, T.; Suzuki, K.; Chichibu, S.; Nakamura, S. Brillouin scattering study of gallium nitride: Elastic stiffness constants. J. Phys. :Condens. Matter 1997, 9, 241-248.

[69] Schwarz, R. B.; Khachaturyan, K.; Weber, E. R. Elastic moduli of gallium nitride. Appl. Phys. Lett. 1997, 70, 1122-1124.

[70] Polian, A.; Grimsditch, M.; Grzegory, I. Elastic constants of gallium nitride. J. Appl. Phys. 1996, 79, 3343-3344.

[71] Kim, K.; Lambrecht, W. R. L.; Segall, B. Electronic structure of $\mathrm{GaN}$ with strain and phonon distortions. Phys. Rev. B 1994, 50, 1502-1505.

[72] Kato, R.; Hama, J. First-principles calculation of the elastic stiffness tensor of aluminium nitride under high pressure. J. Phys. :Condens. Matter 1994, 6, 7617-7632.

[73] Ruiz, E.; Alvarez, S.; Alemany, P. Electronic structure and properties of AlN. Phys. Rev. B 1994, 49, 7115-7123.

[74] McNeil, L. E.; Grimsditch, M.; French, R. H. Vibrational spectroscopy of aluminum nitride. J. Am. Ceram. Soc. 1993, 76, 1132-1136.

[75] Tsubouchi, K.; Mikoshiba, N. Zero-temperature-coefficient SAW devices on AlN epitaxial films. IEEE Trans. Sonics Ultrason. 1985, $32,634-644$.
[76] Savastenko, V. A.; Sheleg, A. U. Study of the elastic properties of gallium nitride. Phys. Status Solidi A 1978, 48, K135-K139.

[77] Shen, X. Q.; Takahashi, T.; Ide, T.; Shimizu, M. Mechanisms of the micro-crack generation in an ultra-thin $\mathrm{AlN} / \mathrm{GaN}$ superlattice structure grown on $\mathrm{Si}(110)$ substrates by metalorganic chemical vapor deposition. J. Appl. Phys. 2015, 118, 125307.

[78] Einfeldt, S.; Heinke, H.; Kirchner, V.; Hommel, D. Strain relaxation in AlGaN/GaN superlattices grown on GaN. J. Appl. Phys. 2001, 89, 2160-2167.

[79] Tripathy, S.; Chua, S. J.; Chen, P.; Miao, Z. L. Micro-Raman investigation of strain in $\mathrm{GaN}$ and $\mathrm{Al}_{x} \mathrm{Ga}_{1-x} \mathrm{~N} / \mathrm{GaN}$ heterostructures grown on Si(111). J. Appl. Phys. 2002, 92, 3503-3510.

[80] Ramkumar, C.; Prokofyeva, T.; Seon, M.; Holtz, M.; Choi, K.; Yun, J.; Nikishin, S. A.; Temkin, H. Micro-Raman scattering from hexagonal $\mathrm{GaN}$, AlN, and $\left.\mathrm{Al}_{x} \mathrm{Ga}_{1-x}\right\} \mathrm{N}$ grown on (111) oriented silicon: Stress mapping of cracks. MRS Online Proc. Libr. 2001, 693, 39-43.

[81] Agrawal, M.; Dharmarasu, N.; Radhakrishnan, K.; Ravikiran, L. Structural properties of GaN grown on AlGaN/AlN stress mitigating layers on $100-\mathrm{mm}$ Si (111) by ammonia molecular beam epitaxy. Thin Solid Films 2012, 520, 7109-7114.

[82] Lee, H. P.; Perozek, J.; Rosario, L. D.; Bayram, C. Investigation of $\mathrm{AlGaN} / \mathrm{GaN}$ high electron mobility transistor structures on $200-\mathrm{mm}$ silicon (111) substrates employing different buffer layer configurations. Sci. Rep. 2016, 6, 37588.

[83] Christy, D.; Watanabe, A.; Egawa, T. Influence of strain induced by AlN nucleation layer on the electrical properties of $\mathrm{AlGaN} / \mathrm{GaN}$ heterostructures on $\mathrm{Si}(111)$ substrate. AIP Adv. 2014, 4, 107104.

[84] Bansal, A.; Wang, K.; Lundh, J. S.; Choi, S.; Redwing, J. M. Effect of Ge doping on growth stress and conductivity in $\mathrm{Al}_{x} \mathrm{Ga}_{1-x} \mathrm{~N}$. Appl. Phys. Lett. 2019, 114, 142101.

[85] Amano, H.; Baines, Y.; Beam, E.; Borga, M.; Bouchet, T.; Chalker, P. R.; Charles, M.; Chen, K. J.; Chowdhury, N.; Chu, R. M. et al. The $2018 \mathrm{GaN}$ power electronics roadmap. J. Phys. D: Appl. Phys. 2018, 51, 163001.

[86] Sun, H. D.; Mitra, S.; Subedi, R. C.; Zhang, Y.; Guo, W.; Ye, J. C.; Shakfa, M. K.; Ng, T. K.; Ooi, B. S.; Roqan, I. S. et al. Unambiguously enhanced ultraviolet luminescence of AlGaN wavy quantum well structures grown on large misoriented sapphire substrate. Adv. Funct. Mater. 2019, 29, 1905445.

[87] Huang, C.; Zhang, H. C.; Sun, H. D. Ultraviolet optoelectronic devices based on AlGaN-SiC platform: Towards monolithic photonics integration system. Nano Energy 2020, 77, 105149.

[88] Zhang, H. C.; Huang, C.; Song, K.; Yu, H. B.; Xing, C.; Wang, D. H.; Liu, Z. L.; Sun, H. D. Compositionally graded III-nitride alloys: Building blocks for efficient ultraviolet optoelectronics and power electronics. Rep. Prog. Phys. 2021, 84, 044401. 